\title{
Honey Sharma
}

\section{On Durrmeyer-type generalization of $(p, q)$-Bernstein operators}

Received: 29 December 2015 / Accepted: 20 July 2016 / Published online: 2 August 2016

(C) The Author(s) 2016. This article is published with open access at Springerlink.com

\begin{abstract}
In this paper, we introduce $(p, q)$-Bernstein Durrmeyer operators. We define $(p, q)$-beta integral and use it to obtain the moments of the operators. We obtain uniform convergence of the operators by using Korovkin's theorem. We estimate direct results of the operators by means of modulus of continuity and Peetre $K$-functional. Finally, we find Voronovskaya-type theorem for the operators.
\end{abstract}

Mathematics Subject Classification $41 \mathrm{~A} 25 \cdot 41 \mathrm{~A} 35$

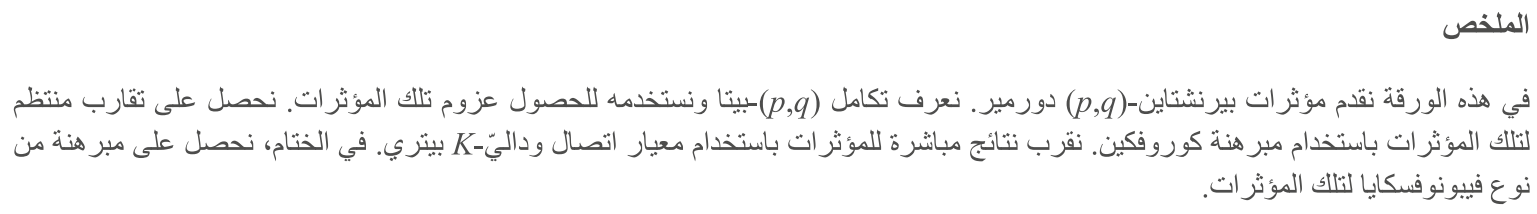

\section{Introduction}

In the last two decades, various mathematicians have introduced $q$-analogues of different discrete and continuous operators and investigated their approximation properties (for a detailed study, see [8]). One may refer to $[11,16]$ to study notation and details of quantum calculus.

We begin by recalling certain notations of $(p, q)$-calculus (for details see $[9,15,22,23]$ ).

Let $0<q<p \leq 1$. The $(p, q)$-integer $[n]_{p, q}$ and $(p, q)$-factorial $[n]_{p, q}$ ! are defined by

$$
\begin{aligned}
{[n]_{p, q} } & =\frac{p^{n}-q^{n}}{p-q} n=0,1,2, \ldots \\
{[n]_{p, q} ! } & = \begin{cases}{[1]_{p, q}[2]_{p, q} \cdots[n]_{p, q},} & n \geq 1 \\
1, & n=0\end{cases}
\end{aligned}
$$

For integers $0 \leq k \leq n,(p, q)$-binomial is defined as

$$
\left[\begin{array}{l}
n \\
k
\end{array}\right]_{p, q}=\frac{[n]_{p, q} !}{[k]_{p, q} ![n-k]_{p, q} !}
$$

H. Sharma $(\varangle)$

Gulzar Group of Institutions, Khanna, Ludhiana, Punjab, India

E-mail: pro.sharma.h@gmail.com 
The $(p, q)$-polynomial expansion is

$$
(x+y)_{p, q}^{n}=(x+y)(p x+q y)\left(p^{2} x+q^{2} y\right) \cdots\left(p^{n-1} x+q^{n-1} y\right) .
$$

Let $f:[0, a] \rightarrow R$. Then $(p, q)$-integration of a function $f$ is defined by,

$$
\int_{0}^{a} f(x) \mathrm{d}_{p, q} x=(p-q) a \sum_{k=0}^{\infty} \frac{q^{k}}{p^{k+1}} f\left(\frac{q^{k}}{p^{k+1}} a\right), \quad \text { when }\left|\frac{p}{q}\right|>1 .
$$

In 2015, Mursaleen et al. [17-21] introduced $(p, q)$-Bernstein operators and its variant. Very recently, Acar [1] introduced $(p, q)$-analogue of Sźasz-Mirakyan operators and after his construction Sharma, Gupta [25] introduced Kantrovich modifications of $(p, q)$-Sźasz-Mirakyan operators.

For $0<q<p \leq 1, n \in \mathbb{N}$ and $f \in C[0,1],(p, q)$-Bernstein operators are defined as:

$$
B_{n}^{(p, q)}(f ; x)=p^{-n(n-1) / 2} \sum_{k=0}^{n} b_{n, k}^{(p, q)}(x) f\left(\frac{p^{-k}[k]_{p, q}}{[n]_{p, q}}\right),
$$

where $b_{n}^{(p, q)}(x)$ is basis of $(p, q)$-Bernstein given as

$$
b_{n, k}^{(p, q)}(x)=p^{k(k-1) / 2}\left[\begin{array}{l}
n \\
k
\end{array}\right]_{p, q} x^{k}(1-x)_{p, q}^{n-k} .
$$

By using identity $\sum_{k=0}^{n} p^{k(k-1) / 2}\left[\begin{array}{l}n \\ k\end{array}\right]_{p, q} x^{k}(1-x)_{p, q}^{n-k}=p^{n(n-1) / 2}$, moments of $(p, q)$-Bernstein operators can be obtained as:

Lemma 1.1 Let $0<q<p \leq 1$ and $n \in \mathbb{N}$. We have

$$
\begin{aligned}
B_{n}^{(p, q)}(1 ; x) & =1 \\
B_{n}^{(p, q)}(t ; x) & =x p^{-n} \\
B_{n}^{(p, q)}\left(t^{2} ; x\right) & =\frac{p^{-n-1}}{[n]_{p, q}} x+\frac{[n-1]_{p, q} p^{-2 n} q}{[n]_{p, q}} x^{2} .
\end{aligned}
$$

Bernstein polynomials, their Durrmeyer variants and Sźasz operators which are generalization of Bernstein polynomials have been studied intensively by many researchers; for details one may refer to $[2-7,13,14,24,26]$. Motivated by these operators, we introduce $(p, q)$-Bernstein Durrmeyer operators for $0<q<p \leq 1, n \in \mathbb{N}$ and $f \in C[0,1]$ as:

$$
D_{n}^{(p, q)}(f ; x)=[n+1]_{p, q} p^{-n^{2}} \sum_{k=0}^{n} b_{n, k}^{(p, q)}(x)\left(\frac{q}{p}\right)^{-k} \int_{0}^{1} b_{n, k}^{(p, q)}(q t) f(t) \mathrm{d}_{p, q} t .
$$

Clearly, for $p>1,(p, q)$-Bernstein Durrmeyer operators coincide with $q$-Bernstein Durrmeyer operators [12].

To obtain the moments of proposed operators, we first define $(p, q)$-beta integral.

Definition 1.2 For $0<q<p \leq 1$ and $s, t \in \mathbb{R}^{+},(p, q)$-beta integral is defined as:

$$
\beta_{p, q}(t, s)=\int_{0}^{1} x^{t-1}(1-q x)_{p, q}^{s-1} \mathrm{~d}_{p, q} x .
$$

In the next lemma, we give relation between $(p, q)$-beta integral and $q$-beta integral. 
Lemma 1.3 For $0<q<p \leq 1$ and $s, t \in \mathbb{R}^{+}$, we have

$$
\beta_{p, q}(t, s)=p^{(s-1)(s-2) / 2-(t-1)} \beta_{q / p}(t, s),
$$

where $\beta_{q / p}(t, s)$ is $q$ / p-analogue of beta function.

Proof First, we show that for $0<q<p \leq 1$

$$
\int_{0}^{a} f(x) \mathrm{d}_{p, q} x=\int_{0}^{a} f(x / p) \mathrm{d}_{q / p} x .
$$

Using definition of $(p, q)$-integration and $q$-integration of a function $f \in C[0, a]$, we have

$$
\begin{aligned}
\int_{0}^{a} f(t) \mathrm{d}_{p, q} t & =(p-q) a \sum_{k=0}^{\infty} \frac{q^{k}}{p^{k+1}} f\left(\frac{q^{k}}{p^{k+1}} a\right) \\
& =(1-q / p) a \sum_{k=0}^{\infty}\left(\frac{q}{p}\right)^{k} f\left(\frac{a}{p}\left(\frac{q}{p}\right)^{k}\right) \\
& =\int_{0}^{a} f(x / p) \mathrm{d}_{q / p} x .
\end{aligned}
$$

By using Equality (1.2) and identity $(1-x)_{p, q}^{n}=p^{n(n-1) / 2}(1-x)_{q / p}^{n}$, we get

$$
\begin{aligned}
\beta_{p, q}(t, s) & =\int_{0}^{1} x^{t-1}(1-q x)_{p, q}^{s-1} \mathrm{~d}_{p, q} x \\
& =\int_{0}^{1}(x / p)^{t-1}(1-q x / p)_{p, q}^{s-1} \mathrm{~d}_{q / p} x \\
& =\int_{0}^{1}(x / p)^{t-1} p^{(s-1)(s-2) / 2}(1-q / p x)_{q / p}^{s-1} \mathrm{~d}_{q / p} x \\
& =p^{(s-1)(s-2) / 2-(t-1)} \beta_{q / p}(t, s) .
\end{aligned}
$$

\section{Moments}

In this section, we obtain the moments for purposed $(p, q)$-Bernstein Durrmeyer operators. We also estimate $m t h$-order moments of the operators and obtain uniform convergence of the operators using Korovkin's type theorem.

Lemma 2.1 For $s=0,1,2,3 \ldots$, we have

$$
\int_{0}^{1} b_{n, k}^{(p, q)}(q t) t^{s} \mathrm{~d}_{p, q} t=\left(\frac{q}{p}\right)^{k} p^{-k s} p^{n(n+2 s+1) / 2} \frac{[n]_{p, q} ![k+s]_{p, q} !}{[k]_{p, q} ![n+s+1]_{p, q} !} .
$$

Proof By Lemma 1.3, we have

$$
\begin{aligned}
\int_{0}^{1} b_{n, k}^{(p, q)}(q t) t^{s} \mathrm{~d}_{p, q} t & =p^{k(k-1) / 2}\left[\begin{array}{l}
n \\
k
\end{array}\right]_{p, q} \int_{0}^{1} t^{s} q^{k} t^{k}(1-q t)_{p, q}^{n-k} \mathrm{~d}_{p, q} t \\
& =p^{k(k-1) / 2}\left[\begin{array}{l}
n \\
k
\end{array}\right]_{p, q} q^{k} \beta_{p, q}(s+k+1, n-k+1) \\
& =p^{k(k-1) / 2}\left[\begin{array}{l}
n \\
k
\end{array}\right]_{p, q} q^{k} p^{(n-k)(n-k-1) / 2-(s+k)} \beta_{q / p}(s+k+1, n-k+1)
\end{aligned}
$$


Using $\beta_{q}(t+1, s+1)=\frac{[t]_{q} ![s]_{q} !}{[s+t+1]_{q} !}$ and $[n]_{p, q} !=p^{n(n-1) / 2}[n]_{q / p}$, we get

$$
\begin{aligned}
\int_{0}^{1} b_{n, k}^{(p, q)}(q t) t^{s} \mathrm{~d}_{p, q} t & =p^{k(k-1) / 2}\left[\begin{array}{l}
n \\
k
\end{array}\right]_{p, q} q^{k} p^{(n-k)(n-k-1) / 2-(s+k)} \frac{[s+k]_{q / p} ![n-k]_{q / p} !}{[n+s+1]_{q / p} !} \\
& =p^{k(k-1) / 2}\left[\begin{array}{l}
n \\
k
\end{array}\right]_{p, q} q^{k} p^{(n+s)(n+s+1) / 2-(s+k)(s+k+1) / 2} \frac{[s+k]_{p, q} ![n-k]_{p, q} !}{[n+s+1]_{p, q} !} \\
& =\left(\frac{q}{p}\right)^{k} p^{-k s} p^{n(n+2 s+1) / 2} \frac{[n]_{p, q} ![k+s]_{p, q} !}{[k]_{p, q} ![n+s+1]_{p, q} !}
\end{aligned}
$$

Lemma 2.2 Let $0<q<p \leq 1$ and $n \in \mathbb{N}$. We have

$$
\begin{aligned}
D_{n}^{(p, q)}(1 ; x) & =1 \\
D_{n}^{(p, q)}(t ; x) & =\frac{1}{[n+2]_{p, q}}\left(p^{n}+q[n]_{p, q} x\right) \\
D_{n}^{(p, q)}\left(t^{2} ; x\right) & =\frac{(p+q) p^{2 n}+(p+q)^{2} q p^{n-1}[n]_{p, q} x+q^{4}[n]_{p, q}[n-1]_{p, q} x^{2}}{[n+2]_{p, q}[n+3]_{p, q}} .
\end{aligned}
$$

Proof Using Lemma 2.1 for $s=0,1,2$, we have

$$
\begin{aligned}
\int_{0}^{1} b_{n, k}^{(p, q)}(q t) \mathrm{d}_{p, q} t & =\left(\frac{q}{p}\right)^{k} p^{n(n+1) / 2} \frac{1}{[n+1]_{p, q}} \\
\int_{0}^{1} t b_{n, k}^{(p, q)}(q t) \mathrm{d}_{p, q} t & =\left(\frac{q}{p}\right)^{k} p^{-k} p^{n(n+3) / 2} \frac{[k+1]_{p, q}}{[n+1]_{p, q}[n+2]_{p, q}} \\
\int_{0}^{1} t^{2} b_{n, k}^{(p, q)}(q t) \mathrm{d}_{p, q} t & =\left(\frac{q}{p}\right)^{k} p^{-2 k} p^{n(n+5) / 2} \frac{[k+1]_{p, q}[k+2]_{p, q}}{[n+1]_{p, q}[n+2]_{p, q}[n+3]_{p, q}} .
\end{aligned}
$$

Using Equality (2.4) and Lemma 1.1, first moment can be found trivially.

Also, by using Equality (2.5), Lemma 1.1 and $[k+1]_{p, q}=p^{k}+q[k]_{p, q}$, we get

$$
\begin{aligned}
D_{n}^{(p, q)}(t ; x) & =[n+1]_{p, q} p^{-n^{2}} \sum_{k=0}^{n} b_{n, k}^{(p, q)}(x) p^{-k} p^{n(n+3) / 2} \frac{[k+1]_{p, q}}{[n+1]_{p, q}[n+2]_{p, q}} \\
& =\frac{1}{[n+2]_{p, q}} p^{\left(-n^{2}+3 n\right) / 2} \sum_{k=0}^{n} b_{n, k}^{(p, q)}(x) p^{-k}\left(p^{k}+q[k]_{p, q}\right) \\
& =\frac{1}{[n+2]_{p, q}} p^{\left(-n^{2}+3 n\right) / 2} \sum_{k=0}^{n} b_{n, k}^{(p, q)}(x)+\frac{[n]_{p, q}}{[n+2]_{p, q}} p^{\left(-n^{2}+3 n\right) / 2} q \sum_{k=0}^{n} b_{n, k}^{(p, q)}(x) p^{-k} \frac{[k]_{p, q}}{[n]_{p, q}} \\
& =\frac{1}{[n+2]_{p, q}} p^{n} B_{n}^{(p, q)}(1 ; x)+\frac{[n]_{p, q}}{[n+2]_{p, q}} p^{n} q B_{n}^{(p, q)}(t ; x) \\
& =\frac{1}{[n+2]_{p, q}} p^{n}+\frac{[n]_{p, q}}{[n+2]_{p, q}} q x \\
& =\frac{1}{[n+2]_{p, q}}\left(p^{n}+q[n]_{p, q} x\right) .
\end{aligned}
$$


Finally, using Equality (2.6) and Lemma 1.1, we have

$$
\begin{aligned}
D_{n}^{(p, q)}\left(t^{2} ; x\right)= & {[n+1]_{p, q} p^{-n^{2}} \sum_{k=0}^{n} b_{n, k}^{(p, q)}(x) p^{-2 k} p^{n(n+5) / 2} \frac{[k+1]_{p, q}[k+2]_{p, q}}{[n+1]_{p, q}[n+2]_{p, q}[n+3]_{p, q}} } \\
= & \frac{1}{[n+2]_{p, q}[n+3]_{p, q}} p^{\left(-n^{2}+5 n\right) / 2} \sum_{k=0}^{n} b_{n, k}^{(p, q)}(x) p^{-2 k}\left(p^{k}+q[k]_{p, q}\right)\left((p+q) p^{k}+q^{2}[k]_{p, q}\right) \\
= & \frac{(p+q)}{[n+2]_{p, q}[n+3]_{p, q}} p^{\left(-n^{2}+5 n\right) / 2} \sum_{k=0}^{n} b_{n, k}^{(p, q)}(x) \\
& +\frac{q(2 q+p)[n]_{p, q}}{[n+2]_{p, q}[n+3]_{p, q}} p^{\left(-n^{2}+5 n\right) / 2} \sum_{k=0}^{n} b_{n, k}^{(p, q)}(x) \frac{p^{-k}[k]_{p, q}}{[n]_{p, q}} \\
& +\frac{q^{3}[n]_{p, q}^{2}}{[n+2]_{p, q}[n+3]_{p, q}} p^{\left(-n^{2}+5 n\right) / 2} \sum_{k=0}^{n} b_{n, k}^{(p, q)}(x)\left(\frac{p^{-k}[k]_{p, q}}{[n]_{p, q}}\right) \\
= & \frac{1}{[n+2]_{p, q}[n+3]_{p, q}} p^{2 n} \\
& \times\left((p+q) B_{n}^{(p, q)}(1 ; x)+q(2 q+p)[n]_{p, q} B_{n}^{(p, q)}(t ; x)+q^{3}[n]_{p, q}^{2} B_{n}^{(p, q)}\left(t^{2} ; x\right)\right) \\
= & \frac{1}{[n+2]_{p, q}[n+3]_{p, q}} \\
& \times\left((p+q) p^{2 n}+q(2 q+p) p^{n}[n]_{p, q} x+q^{3}[n]_{p, q}\left(p^{n-1} x+[n-1]_{p, q} q x^{2}\right)\right) \\
= & \frac{(p+q) p^{2 n}+(p+q)^{2} q p^{n-1}[n]_{p, q} x+q^{4}[n]_{p, q}[n-1]_{p, q} x^{2}}{[n+2]_{p, q}[n+3]_{p, q}}
\end{aligned}
$$

Remark 2.3 For $p=1$, the above moments coincide with moments of $q$-Durrmeyer operators due to Gupta [12].

Remark 2.4 Central moments of $(p, q)$-Durrmeyer can be obtained as

$$
\begin{aligned}
D_{n}^{(p, q)}(t-x ; x)= & \frac{p^{n}+\left(q[n]_{p, q}-[n+2]_{p, q}\right) x}{[n+2]_{p, q}} \\
D_{n}^{(p, q)}\left((t-x)^{2} ; x\right)= & \frac{\left(q^{4}[n]_{p, q}[n-1]_{p, q}-2 q[n]_{p, q}[n+3]_{p, q}+[n+2]_{p, q}[n+3]_{p, q}\right) x^{2}}{[n+2]_{p, q}[n+3]_{p, q}} \\
& +\frac{\left((p+q)^{2} q p^{n-1}[n]_{p, q}-2 p^{n}[n+3]_{p, q}\right) x+(q+p) p^{2 n}}{[n+2]_{p, q}[n+3]_{p, q}} .
\end{aligned}
$$

Remark 2.5 For $q \in(0,1)$ and $p \in(q, 1]$, by simple computations, $\lim _{n \rightarrow \infty}[n]_{p, q}=1 /(p-q)$. In order to obtain results for order of convergence of the operators, we take $q_{n} \in(0,1), p_{n} \in\left(q_{n}, 1\right]$ such that $\lim _{n \rightarrow \infty} p_{n}=1$ and $\lim _{n \rightarrow \infty} q_{n}=1$, so that $\lim _{n \rightarrow \infty} \frac{1}{[n]_{p_{n}, q_{n}}}=0$. Such a sequence can always be constructed, for example, we can take $q_{n}=1-1 / n$ and $p_{n}=1-1 / 2 n$, clearly $\lim _{n \rightarrow \infty} p_{n}^{n}=e^{-1 / 2}, \lim _{n \rightarrow \infty} q_{n}^{n}=e^{-1}$ and $\lim _{n \rightarrow \infty} \frac{1}{[n]_{p_{n}, q_{n}}}=0$.

Remark 2.6 Let $\left(p_{n}\right)_{n}$ and $\left(q_{n}\right)_{n}$ be sequences as defined in Remark 2.5 and $\lim _{n \rightarrow \infty} p_{n}^{n}=a, \lim _{n \rightarrow \infty} p_{n}^{n}=b$ for $0 \leq a, b<1$. We have

$$
\begin{gathered}
\lim _{n \rightarrow \infty}[n]_{p_{n}, q_{n}} D_{n}^{(p, q)}(t-x ; x)=a+\alpha x, \\
\lim _{n \rightarrow \infty}[n]_{p_{n}, q_{n}} D_{n}^{(p, q)}\left((t-x)^{2} ; x\right)=x(\gamma x+2 a),
\end{gathered}
$$

where $\alpha=\lim _{n \rightarrow \infty}[n]_{p_{n}, q_{n}}\left(q_{n}-1\right)$ and $\gamma=\lim _{n \rightarrow \infty}[n]_{p_{n}, q_{n}}\left(q_{n}^{4}-2 q_{n}+1\right)$. 
Theorem 2.7 For $s>0$, sth order moment of $(p, q)$-Durrmeyer operators are given by

$$
D_{n}^{(p, q)}\left(t^{s} ; x\right)=\frac{[n+1]_{p, q} !}{[n+s+1]_{p, q} !} p^{n s} \sum_{j=0}^{s} C_{j}(s, p, q)[n]_{p, q}^{j} B_{n}^{(p, q)}\left(t^{j} ; x\right),
$$

where $C_{j}(s, p, q)$ for $j=0,1,2, \ldots, s$ are constants.

Proof For $s>0$, using definition of operators and Lemma 2.1

$$
\begin{aligned}
D_{n}^{(p, q)}\left(t^{s} ; x\right) & =[n+1]_{p, q} p^{-n^{2}} \sum_{k=0}^{n} b_{n, k}^{(p, q)}(x) p^{-k s} p^{n(n+2 s+1) / 2} \frac{[n]_{p, q} ![k+s]_{p, q} !}{[k]_{p, q} ![n+s+1]_{p, q} !} \\
& =\frac{[n+1]_{p, q} !}{[n+s+1]_{p, q} !} p^{-n(n-1) / 2} p^{n s} \sum_{k=0}^{n} b_{n, k}^{(p, q)}(x) p^{-k s}[k+1]_{p, q}[k+2]_{p, q} \cdots[k+s]_{p, q} .
\end{aligned}
$$

Using equality $[k+j]_{p, q}=p^{k}[j]_{p, q}+q^{j}[k]_{p, q}$, we have

$$
\begin{aligned}
{[k+1]_{p, q}[k+2]_{p, q} \cdots[k+s]_{p, q} } & =\prod_{j=1}^{s}\left(p^{k}[j]_{p, q}+q^{j}[k]_{p, q}\right) \\
& =\sum_{j=0}^{s} C_{j}(s, p, q) p^{k(s-j)}[k]_{p, q}^{j},
\end{aligned}
$$

where $C_{j}(s, p, q)$ for $j=0,1,2, \ldots, s$ are constants. Finally, using above equality, we get

$$
\begin{aligned}
D_{n}^{(p, q)}\left(t^{s} ; x\right) & =\frac{[n+1]_{p, q} !}{[n+s+1]_{p, q} !} p^{-n(n-1) / 2} p^{n s} \sum_{k=0}^{n} b_{n, k}^{(p, q)}(x) p^{-k s} \sum_{j=0}^{s} C_{j}(s, p, q) p^{k(s-j)}[k]_{p, q}^{j} \\
& =\frac{[n+1]_{p, q} !}{[n+s+1]_{p, q} !} p^{n s} \sum_{j=0}^{s} C_{j}(s, p, q)[n]_{p, q}^{j} p^{-n(n-1) / 2} \sum_{k=0}^{n} b_{n, k}^{(p, q)}(x)\left(\frac{p^{-k}[k]_{p, q}}{[n]_{p, q}}\right)^{j} \\
& =\frac{[n+1]_{p, q} !}{[n+s+1]_{p, q} !} p^{n s} \sum_{j=0}^{s} C_{j}(s, p, q)[n]_{p, q}^{j} B_{n}^{(p, q)}\left(t^{j} ; x\right) .
\end{aligned}
$$

Theorem 2.8 Let $\left(p_{n}\right)_{n}$ and $\left(q_{n}\right)_{n}$ be sequences as defined in Remark 2.5. Then for each $f \in C[0,1]$, $D_{n}^{\left(p_{n}, q_{n}\right)}(f ;$.$) converges uniformly to f$.

Proof By Korovkin theorem, it is sufficient to show that $\lim _{n \rightarrow \infty}\left\|D_{n}^{\left(p_{n}, q_{n}\right)}\left(e_{m} ;.\right)-e_{m}\right\|=0$ for $e_{m}=t^{m}$, $m=0,1,2$.

Using Eq. (2.1), result for $m=0$ is trivial.

For $m=1$, result is obtained using Eq. (2.2), as follows:

$$
\begin{aligned}
\lim _{n \rightarrow \infty}\left\|D_{n}^{\left(p_{n}, q_{n}\right)}\left(e_{1} ; .\right)-e_{1}\right\| & \leq \lim _{n \rightarrow \infty}\left|\frac{p_{n}^{n}}{[n+2]_{p_{n}, q_{n}}}\right|+\lim _{n \rightarrow \infty}\left|\frac{\left(q_{n}[n]_{p_{n}, q_{n}}-[n+2]_{p_{n}, q_{n}}\right)}{[n+2]_{p_{n}, q_{n}}}\right| \\
& =0 .
\end{aligned}
$$

Finally, using Eq. (2.3), we get

$$
\begin{aligned}
\lim _{n \rightarrow \infty}\left\|D_{n}^{\left(p_{n}, q_{n}\right)}\left(e_{2} ; .\right)-e_{2}\right\| \leq & \lim _{n \rightarrow \infty}\left|\frac{\left(p_{n}+q_{n}\right) p_{n}^{2 n}}{[n+2]_{p_{n}, q_{n}}[n+3]_{p_{n}, q_{n}}}\right| \\
& +\lim _{n \rightarrow \infty}\left|\frac{\left(p_{n}+q_{n}\right)^{2} q_{n} p_{n}^{n-1}[n]_{p_{n}, q_{n}}}{[n+2]_{p_{n}, q_{n}}[n+3]_{p_{n}, q_{n}}}\right|+\lim _{n \rightarrow \infty}\left|\frac{q_{n}^{4}[n]_{p_{n}, q_{n}}[n-1]_{p_{n}, q_{n}}}{[n+2]_{p_{n}, q_{n}}[n+3]_{p_{n}, q_{n}}}-1\right| \\
= & 0 .
\end{aligned}
$$

The proof is now complete. 


\section{Main theorems}

For $f \in C[0,1]$ modulus of continuity $\omega(f, \delta)$ for $\delta>0$ is defined as

$$
\omega(f, \delta)=\sup _{|x-y| \leq \delta ; x, y \in[0,1]}|f(x)-f(y)|
$$

and modulus of continuity of second order is defined as

$$
\omega_{2}(f, \delta)=\sup _{|h| \leq \delta ; x, x+h, x+2 h \in[0,1]}|f(x+2 h)-2 f(x+h)+f(x)| .
$$

Peetre's $K$-functional is defined by

$$
K_{2}(f, \delta)=\inf _{g \in W^{2}}\left\{\|f-g\|+\delta\left\|g^{\prime \prime}\right\|\right\},
$$

where $W^{2}=\left\{g \in C[0,1]: g^{\prime}, g^{\prime \prime} \in C[0,1]\right\}$. By [10, p. 177, Theorem 2.4], there exists a positive constant $C>0$ such that $K_{2}(f, \delta) \leq C \omega_{2}(f, \sqrt{\delta}), \delta>0$.

Theorem 3.1 For $f \in C[0,1]$,

$$
\left|D_{n}^{\left(p_{n}, q_{n}\right)}(f(t)-f(x) ; x)\right| \leq 2 \omega\left(f, \eta_{n}(x)\right),
$$

where $\eta_{n}(x)=\sqrt{D_{n}^{\left(p_{n}, q_{n}\right)}\left((t-x)^{2} ; x\right)}$.

Proof By linearity and monotonicity of operators, we get

$$
\left|D_{n}^{\left(p_{n}, q_{n}\right)}(f(t)-f(x) ; x)\right| \leq D_{n}^{\left(p_{n}, q_{n}\right)}(|f(t)-f(x)| ; x) .
$$

By using property of modulus of continuity $|f(t)-f(x)| \leq \omega\left(f, \eta_{n}\right)\left(1+\frac{|t-x|}{\eta_{n}}\right)$, we get

$$
\left|D_{n}^{\left(p_{n}, q_{n}\right)}(f(t)-f(x) ; x)\right| \leq \omega\left(f, \eta_{n}\right)\left(1+\frac{1}{\eta_{n}} \sqrt{D_{n}^{\left(p_{n}, q_{n}\right)}\left((t-x)^{2} ; x\right)}\right),
$$

taking $\eta_{n}=\sqrt{D_{n}^{\left(p_{n}, q_{n}\right)}\left((t-x)^{2} ; x\right)}$, we finally get result.

Theorem 3.2 Let $\left(p_{n}\right)_{n}$ and $\left(q_{n}\right)_{n}$ be sequences as defined in Remark 2.5. Let $f \in C[0,1]$. Then for all $n \in \mathbb{N}$, there exists an absolute constant $C>0$ such that

$$
\left|D_{n}^{\left(p_{n}, q_{n}\right)}(f ; x)-f(x)\right| \leq C \omega_{2}\left(f, \delta_{n}(x)\right)+\omega\left(f, \alpha_{n}(x)\right),
$$

where $\delta_{n}(x)=\left\{D_{n}^{(p, q)}\left((t-x)^{2} ; x\right)+\left(D_{n}^{(p, q)}(t-x ; x)\right)^{2}\right\}^{\frac{1}{2}}$ and $\alpha_{n}(x)=D_{n}^{(p, q)}(t-x ; x)$.

Proof For $x \in[0,1]$, we consider the operators $D_{n}^{*}(f ; x)$ as

$$
D_{n}^{*}(f ; x)=D_{n}^{\left(p_{n}, q_{n}\right)}(f ; x)+f(x)-f\left(\frac{p_{n}^{n}+q_{n}[n]_{p_{n}, q_{n}} x}{[n+2]_{p_{n}, q_{n}}}\right) .
$$

Using above operators and Eq. (2.2), we immediately get $D_{n}^{*}(t-x ; x)=0$. For $x \in[0,1]$ and $g \in W^{2}$ using the Taylor's formula, we have

$$
g(t)=g(x)+g^{\prime}(x)(t-x)+\int_{x}^{t}(t-u) g^{\prime \prime}(u) \mathrm{d} u .
$$

Therefore,

$$
\begin{aligned}
D_{n}^{*}(g ; x)-g(x)= & g^{\prime}(x) D_{n}^{*}((t-x) ; x)+D_{n}^{*}\left(\int_{x}^{t}(t-u) g^{\prime \prime}(u) \mathrm{d} u ; x\right) \\
= & D_{n}^{\left(p_{n}, q_{n}\right)}\left(\int_{x}^{t}(t-u) g^{\prime \prime}(u) \mathrm{d} u ; x\right) \\
& -\int_{x}^{\frac{p_{n}^{n}+q_{n}[n] p_{n}, q_{n} x}{[n+2] p_{n}, q_{n}}}\left(\frac{p_{n}^{n}+q_{n}[n]_{p_{n}, q_{n}} x}{[n+2]_{p_{n}, q_{n}}}-u\right) g^{\prime \prime}(u) \mathrm{d} u .
\end{aligned}
$$


Finally, we have

$$
\begin{aligned}
\left|D_{n}^{*}(g ; x)-g(x)\right| \leq & \left|D_{n}^{\left(p_{n}, q_{n}\right)}\left(\int_{x}^{t}(t-u) g^{\prime \prime}(u) \mathrm{d} u ; x\right)\right| \\
& +\left|\int_{x}^{\frac{p_{n}^{n}+q_{n}[n] p_{n}, q_{n} x}{[n+2] p_{n}, q_{n}}}\left(\frac{p_{n}^{n}+q_{n}[n]_{p_{n}, q_{n}} x}{[n+2]_{p_{n}, q_{n}}}-u\right) g^{\prime \prime}(u) \mathrm{d} u\right| \\
\leq & \left\|g^{\prime \prime}\right\| D_{n}^{\left(p_{n}, q_{n}\right)}\left((t-x)^{2} ; x\right)+\left(\frac{p_{n}^{n}+q_{n}[n]_{p_{n}, q_{n}} x}{[n+2]_{p_{n}, q_{n}}}-x\right)^{2}\left\|g^{\prime \prime}\right\| \\
& =\delta_{n}^{2}(x)\left\|g^{\prime \prime}\right\| .
\end{aligned}
$$

Also, we have

$$
\left|D_{n}^{*}(f ; x)\right| \leq\left|D_{n}^{\left(p_{n}, q_{n}\right)}(f ; x)\right|+2\|f\| \leq 3\|f\|
$$

Therefore,

$$
\begin{aligned}
\left|D_{n}^{\left(p_{n}, q_{n}\right)}(f ; x)-f(x)\right| \leq & \left|D_{n}^{*}(f-g ; x)-(f-g)(x)\right|+\left|f\left(\frac{p_{n}^{n}+q_{n}[n]_{p_{n}, q_{n}} x}{[n+2]_{p_{n}, q_{n}}}\right)-f(x)\right| \\
& +\left|D_{n}^{*}(g ; x)-g(x)\right| \\
\leq & \left|D_{n}^{*}(f-g ; x)\right|+|(f-g)(x)|+\left|f\left(\frac{p_{n}^{n}+q_{n}[n]_{p_{n}, q_{n}} x}{[n+2]_{p_{n}, q_{n}}}\right)-f(x)\right| \\
& +\left|D_{n}^{*}(g ; x)-g(x)\right| \\
\leq & 4\|f-g\|+\omega\left(f ;\left|\frac{q_{n}^{2}}{[n]_{p_{n}, q_{n}}\left(p_{n}+q_{n}\right)}+\left(q_{n}-1\right) x\right|\right)+\delta_{n}^{2}(x)\left\|g^{\prime \prime}\right\| .
\end{aligned}
$$

On taking infimum on right-hand side over all $g \in W^{2}$ and by definition of $K$-functional, we get

$$
\left|D_{n}^{\left(p_{n}, q_{n}\right)}(f ; x)-f(x)\right| \leq 4 K_{2}\left(f, \delta_{n}^{2}(x)\right)+\omega\left(f, \alpha_{n}(x)\right) .
$$

Finally, using property $K_{2}(f, \delta) \leq C \omega_{2}(f, \sqrt{\delta})$, we get the result.

Here, we give Voronovskaya-type theorem for the operators.

Theorem 3.3 Let $\left(p_{n}\right)_{n}$ and $\left(q_{n}\right)_{n}$ be sequences as defined in Remark $2.5, \lim _{n \rightarrow \infty} p_{n}^{n}=$ a and $\lim _{n \rightarrow \infty} p_{n}^{n}=$ $b$ for $0 \leq a, b<1$. Then for $f \in C[0,1]$, such that $f^{\prime}, f^{\prime \prime} \in C[0,1]$, we have

$$
\lim _{n \rightarrow \infty}[n]_{p_{n}, q_{n}}\left|D_{n}^{\left(p_{n}, q_{n}\right)}(f ; x)-f(x)\right|=(\alpha x+a) f^{\prime}(x)+x(\gamma x+2 a) f^{\prime \prime}(x) / 2
$$

uniformly on $[0,1]$. Here, $\alpha=\lim _{n \rightarrow \infty}[n]_{p_{n}, q_{n}}\left(q_{n}-1\right)$ and $\gamma=\lim _{n \rightarrow \infty}[n]_{p_{n}, q_{n}}\left(q_{n}^{4}-2 q_{n}+1\right)$.

Proof Using the Taylor's formula for $f \in C[0,1]$,

$$
f(t)=f(x)+f^{\prime}(x)(t-x)+\frac{1}{2} f^{\prime \prime}(x)(t-x)^{2}+r(t, x)(t-x)^{2},
$$

where $r(t, x)$ is reminder term such that $\lim _{t \rightarrow x} r(t, x)=0$. Therefore, we have

$$
\begin{aligned}
{[n]_{p_{n}, q_{n}}\left(D_{n}^{\left(p_{n}, q_{n}\right)}(f ; x)-f(x)\right)=} & {[n]_{p_{n}, q_{n}} f^{\prime}(x) D_{n}^{\left(p_{n}, q_{n}\right)}((t-x) ; x) } \\
& +[n]_{p_{n}, q_{n}} \frac{f^{\prime \prime}(x)}{2} D_{n}^{\left(p_{n}, q_{n}\right)}\left((t-x)^{2} ; x\right) \\
& +[n]_{p_{n}, q_{n}} D_{n}^{\left(p_{n}, q_{n}\right)}\left(r(t, x)(t-x)^{2} ; x\right) .
\end{aligned}
$$

Using Cauchy-Schwartz inequality, we have

$$
D_{n}^{\left(p_{n}, q_{n}\right)}\left(r(t, x)(t-x)^{2} ; x\right) \leq \sqrt{D_{n}^{\left(p_{n}, q_{n}\right)}\left(r^{2}(t, x) ; x\right)} \sqrt{D_{n}^{\left(p_{n}, q_{n}\right)}\left((t-x)^{4} ; x\right)} .
$$


As $r(t, x) \in C[0,1]$, therefore by uniform convergence of operators and fact that $\lim _{t \rightarrow x} r(t, x)=0$, we get

$$
\lim _{n \rightarrow \infty} D_{n}^{\left(p_{n}, q_{n}\right)}\left(r^{2}(t, x) ; x\right)=r^{2}(x, x)=0,
$$

uniformly for any $x \in[0,1]$. Hence, by using above equality and positivity of linear operators, we have

$$
\lim _{n \rightarrow \infty}[n]_{p_{n}, q_{n}} D_{n}^{\left(p_{n}, q_{n}\right)}\left(r(t, x)(t-x)^{2} ; x\right)=0 .
$$

Finally,

$$
\begin{aligned}
\lim _{n \rightarrow \infty}[n]_{p_{n}, q_{n}}\left(D_{n}^{\left(p_{n}, q_{n}\right)}(f ; x)-f(x)\right)= & \lim _{n \rightarrow \infty}[n]_{p_{n}, q_{n}} f^{\prime}(x) D_{n}^{\left(p_{n}, q_{n}\right)}((t-x) ; x) \\
& +\lim _{n \rightarrow \infty}[n]_{p_{n}, q_{n}} \frac{f^{\prime \prime}(x)}{2} D_{n}^{\left(p_{n}, q_{n}\right)}\left((t-x)^{2} ; x\right) .
\end{aligned}
$$

By using Remark 2.6, we get the theorem.

Open Access This article is distributed under the terms of the Creative Commons Attribution 4.0 International License (http:// creativecommons.org/licenses/by/4.0/), which permits unrestricted use, distribution, and reproduction in any medium, provided you give appropriate credit to the original author(s) and the source, provide a link to the Creative Commons license, and indicate if changes were made.

\section{References}

1. Acar, T.: $(p, q)$-Generalization of Sźasz-Mirakyan operators. Math. Methods Appl. Sci. 39(10), 2685-2695 (2016)

2. Acar, T.: Asymptotic formulas for generalized Sźasz-Mirakyan operators. Appl. Math. Comput. 263, 223-239 (2015)

3. Acar, T.; Aral, A.: On pointwise convergence of $q$-Bernstein operators and their $q$-derivatives. Numer. Funct. Anal. Optim. 36(3), 287-304 (2015)

4. Acar, T.; Aral, A.; Gupta, V.: On approximation properties of a new type Bernstein-Durrmeyer operators. Math. Slovaca 65(5), 1107-1122 (2015)

5. Acar, T.; Aral, A.; Mohiuddine, S.A.: On Kantorovich modification of $(p, q)$-Baskakov operators. J. Inequal. Appl. 2016, 98 (2016). doi:10.1186/s13660-016-1045-9

6. Acar, T.; Gupta, V.; Aral, A.: Rate of convergence for generalized Sźasz operators. Bull. Math. Sci. 1(1), 99-113 (2011)

7. Acar, T.; Ulusoy, G.: Approximation by modified Sźasz-Durrmeyer operators. Periodica Mathematica Hungarica 72(1), 64-75 (2016)

8. Aral, A.; Gupta, V.; Agarwal, R.P.: Applications of $q$-Calculus in Operator Theory. Springer, Berlin (2013)

9. Chakrabarti, R.; Jagannathan, R.: A $(p, q)$-oscillator realization of two parameter quantum algebras. J. Phys. A: Math. Gen. 24, 711-718 (1991)

10. DeVore, R.A.; Lorentz, G.G.: Constructive Approximation. Springer, Berlin (1993)

11. Ernst, T.: The history of $q$-calculus and a new method, U.U.D.M. Report 16. Department of Mathematics, Uppsala University, Uppsala (2000)

12. Gupta, V.: Some approximation properties on $q$-Durrmeyer operators. Appl. Math. Comput. 197(1), 172-178 (2008)

13. Gupta, V.; Sharma, H.: Recurrence formula and better approximation for $q$-Durrmeyer operators. Lobachevskii J. Math. 32(2), 140-145 (2011). doi:10.1134/S1995080211020065

14. Gupta, V.; Sharma, H.; Kim, T.; Lee, S.: Properties of $q$-analogue of Beta operator. Adv. Differ. Equ. 86(1), 1-16 (2012)

15. Jagannathan, R.; Rao, K.S.: Two-parameter quantum algebras, twin-basic numbers and associated generalized hypergeometric series. In: Proceedings of the International Conference on Number Theory and Mathematical Physics, December 2005, 20-21

16. Kac, V.; Cheung, P.: Quantum Calculus. Universitext. Springer, New York (2002)

17. Mursaleen, M.; Ansari, K.J., Khan, A.: On ( $p, q)$-analogue of Bernstein operators (2015). arXiv:1503.07404v1

18. Mursaleen, M.; Ansari, K.J.; Khan, A.: Some approximation results by $(p, q)$-analogue of Bernstein-Stancu operators. Appl. Math. Comput. 264, 392-402 (2015)

19. Mursaleen, M.; Ansari, K.J.; Khan, A.: Corrigendum to: "Some approximation results by $(p, q)$-analogue of BernsteinStancu operators" [Appl. Math. Comput. 264 (2015) 392-402]. Appl. Math. Comput. 269, 744-746 (2015)

20. Mursaleen, M.; Ansari, K.J.; Khan, A.: Some approximation results on Bernstein-Schurer operators defined by $(p, q)$ integers. J. Inequal. Appl. 2015, 249 (2015)

21. Mursaleen, M.; Ansari, K.J.; Khan, A.: Corrigendum to: Erratum to "On $(p, q)$-analogue of Bernstein operators" [Appl. Math. Comput. 266 (2015) 874-882]. Appl. Math. Comput. 278, 70-71 (2015)

22. Sadjang, P.N.: On the fundamental theorem of $(p, q)$-calculus and some $(p, q)$-Taylor formulas (2013). arXiv:1309.3934 [math.QA]

23. Sahai, V.; Yadav, S.: Representations of two parameter quantum algebras and $(p, q)$-special functions. J. Math. Anal. Appl. 335, 268-279 (2007) 
24. Sharma, H.: Note on approximation properties of generalized Durrmeyer operators. Math. Sci. 6(24) (2012). doi:10.1186/ 2251-7456-6-24

25. Sharma, H.; Gupta, C.: On ( $p, q)$-generalization of Szász-Mirakyan Kantorovich operators. Bollettino dell’Unione Matematica Italiana. doi:10.1007/s40574-015-0038-9

26. Ulusoy, G.; Acar, T.: $q$-Voronovskaya type theorems for $q$-Baskakov operators. Math. Methods Appl. Sci. 39(12), 3391-3401 (2016) 